\title{
Stimulated Brillouin Scattering in Optical Fibers for Optical Communication Systems
}

\author{
N. A. B. Ahmad ${ }^{1,2}$, S. H. Dahlan ${ }^{1,2}$ and N. A. Cholan ${ }^{1,2+}$ \\ ${ }^{1}$ Research Center for Applied Electromagnetics, Universiti Tun Hussein Onn Malaysia (UTHM), 86400 \\ Parit Raja Batu Pahat, Johor, Malaysia. \\ ${ }^{2}$ Faculty of Electrical and Electronics Engineering, Universiti Tun Hussein Onn Malaysia (UTHM), 86400 \\ Parit Raja Batu Pahat, Johor, Malaysia.
}

\begin{abstract}
This paper investigates stimulated Brillouin scattering (SBS) behaviour and phenomenon by using the theory derived from the two-coupled equation which is frequently used to explain the mutual interaction of the pump wave, the acoustic wave and the Stokes wave. Results show that once the Brillouin threshold is reached, a backward-propagating Stokes wave carries most of the input power. In addition, for a $25 \mathrm{~km}$ single-mode fiber (SMF), it is found that $35 \%$ of the pump power is transferred to the Stokes wave and the power transfer occurs within the first $2.5 \mathrm{~km}$ of the $25 \mathrm{~km}$ SMF.
\end{abstract}

Keywords: Optical communication systems, multiwavelength Fiber lasers, optics nonlinearities, stimulated Brillouin scattering.

\section{Introduction}

Nonlinearities in optical fibers are harmful for optical communication systems as they can limit the transmission power and distort signals in the data transmission [1]. Stimulated Brillouin Scattering (SBS) is one of the prominent nonlinearities that occur in optical fibers especially when the input signal power is relatively high. Due to the input light being converted into the backward direction, the usable optical power level is limited when such SBS occurs, resulting in additional nonlinear losses [2]. Despite the setback, SBS is useful for developing multiwavelength fiber lasers that can function as light sources for optical communication systems. An example of technology that can exploit SBS for being lasers is multiwavelength Brillouin-erbium fiber lasers (BEFLs) [3]-[7]. In BEFLs, gain mechanism of SBS and erbium-doped fiber amplifier (EDFA) are combined such that oscillating signals have enough energy to overcome losses in the laser cavity.

When a wave is incident on an optical fiber, much power is reflected in the backward direction in the form of a Stoke wave due to SBS process. However, not all power is reflected; there is some power transmitted in the same direction as the wave. In BEFLs, this residual power is recycled back into the laser cavity in order to enhance the number of laser lines [5]-[7]. In this paper, we extend such work in understanding the dynamic behaviour of SBS. It is found that, for a $25 \mathrm{~km}$ single-mode fiber (SMF), $35 \%$ of the pump power is transferred to the Stokes wave and the power transfer occurs within the first $2.5 \mathrm{~km}$ of the $25 \mathrm{~km} \mathrm{SMF.}$

\section{Theoretical and Operating Principle}

Fig. 1 shows the process of how the forward (transmitted) and backward (reflected) is measured. When a pump wave with the optical power $P_{P}(0)$ at a certain wavelength $\lambda_{P}$ propagates along an optical fiber, it

+ Corresponding author. Tel.: + (607-4537528)

E-mail address: (noran@uthm.edu.my). 
interacts with the fiber material and creates an acoustic wave at another wavelength $\lambda_{a}$ until it reach the end of the fiber, $P_{P}(z)$. Then, a Stokes wave with the Stokes power, $P_{S}(0)$ at a wavelength $\lambda_{S}=\lambda_{P}-\lambda_{a}$ is generated due to the mutual interaction between the original pump wave, the Stokes wave and the acoustic wave where the three-wave mixing process occurs. The initial growth of stokes wave under continuous wave (CW) and quasi-CW condition can be described by a coupled equation [8]

$$
\begin{aligned}
& \frac{d P_{P}}{d z}=-g_{B} P_{P} P_{S}-\alpha P_{P} \\
& \frac{d P_{S}}{d z}=-g_{B} P_{P} P_{S}+\alpha P_{S}
\end{aligned}
$$

where the negative sign indicates the backward propagation of the Stokes wave and $g_{B}$ is the peak value of the Brillouin gain. The Brillouin scattering produce photons within the bandwidth of Brillouin gain spectrum and hence all frequency components of the wave will be subjected to amplification. The frequency component for which $g_{B}$ is a maximum develops rapidly and normally the development is exponential. For pure silica, $g_{B}$ is a maximum for frequency component which is downshifted from the pump frequency by around $10 \mathrm{GHz}$.

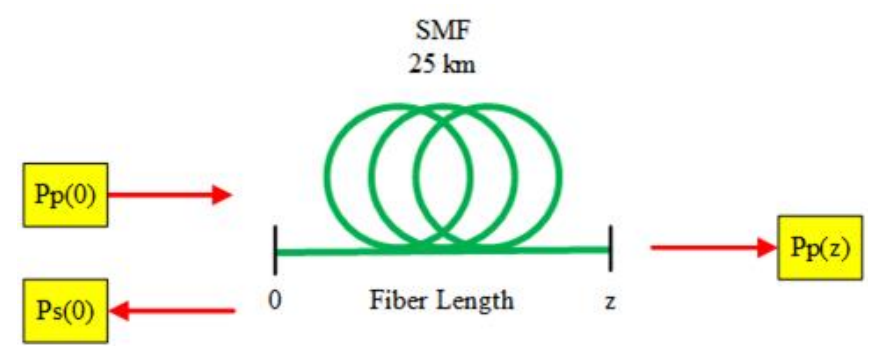

Fig. 1: Illustration of the numerical analysis in an optical fiber

In the absence of fiber losses $(\alpha=0)$, Equations (1) and (2) can be reduced to [8]

$$
P_{S}-P_{P}=C
$$

as $C$ denotes constant. Equation (3) describes the conservation phenomenon on light energy during the Brillouin process. In a situation, where the Stokes power is much smaller than the pump power, it can be assumed that pump power is not depleted and hence the term $-g_{B} P_{P} P_{S}$ in Equation (1) can be neglected. Thus, Equation (1) can be written as

$$
\frac{d P_{P}}{d z}=-\alpha P_{P}
$$

The solution of Equation (4) is given by

$$
P_{P}(z)=P_{P}(0) \exp (-\alpha z),
$$

where $P_{P}(z)$ and $P_{P}(0)$ are pump power at length $\mathrm{z}$ and at $z=0$, respectively. From Equations (2) and (5), the Stokes power is found to grow exponentially in the backward direction as

$$
P_{S}(0)=P_{S}(z) \exp (-\alpha L) \exp \left(g_{B} P_{P}(0) L_{e f f} / A_{e f f}\right),
$$

where $L_{\text {eff }}=\alpha^{-1}[1-\exp (-\alpha L)]$ is the effective length of interaction. The length of $L_{\text {eff }}$ is slightly less than the fiber length, $L$ because of pump absorption.

The pump power at which the Stokes wave power increases significantly and may even be comparable with the pump power is called the threshold power or also known as the SBS threshold power [8]. The SBS threshold power can be obtained from Equations (5) and (6) where it is approximated as

$$
P_{t h} \cong \frac{21 b A_{e f f}}{g_{B} L_{e f f}}
$$


Here, the numerical factor of 21 is only an approximate value as it depends on the exact value of Brillouin gain linewidth [8]. The value of parameters used in the simulation is listed in Table 1. The low value of threshold level makes the SBS process a dominant nonlinear process in silica based optical fibers. Typically, the value of polarization factor $b$ lies between 1 to 2 depending on the relative polarization of pump and Stokes waves. For instance, the $P_{t h}$ will become doubled if polarization factor is 2 . In addition, the fiber inhomogeneous will affect $g_{B}$ and subsequently the $P_{t h}$ since $P_{t h}$ is mainly dependent on $g_{B}$ as portrayed in Equation (7). To some extent, the variation in doping affects the SBS threshold power as well.

Table 1: Basic Configuration Used in Calculation

\begin{tabular}{|l|c|c|}
\hline \multicolumn{1}{|c|}{ Parameter } & Unit & Value \\
\hline Fiber-length $(L)$ & $\mathrm{km}$ & 25 \\
\hline Attenuation $(\alpha)$ & $\mathrm{dB} / \mathrm{km}$ & 0.2 \\
\hline Effective area $\left(A_{\text {eff }}\right)$ & $\mu \mathrm{m}^{2}$ & 85 \\
\hline Brillouin gain $\left(g_{B}\right)$ & $\mathrm{mW}^{-1}$ & $4 \times 10^{-11}$ \\
\hline Polarization effect $(b)$ & - & 1.2 \\
\hline
\end{tabular}

\section{Results and Discussion}

Fig. 2 shows the transmitted and reflected powers as a function of the input power launched into the optical fiber. We plot the graph by solving coupled equations (1) and (2) via numerical method. It is found that the output power for the forward direction measured at $P_{P}(z)$ is linearly related to the launched input power up to $2 \mathrm{~mW}$. However, at the input power higher than $2 \mathrm{~mW}$, the output power became nonlinear where the transmitted power decrease because of pump depletion, and reach a saturation level of $0.7 \mathrm{~mW}$. On the other hand, the reflected signal represented by $P_{S}(0)$ is measured in the backward direction of the fiber due to the backward propagation of SBS. Fig. 2 demonstrates a significant increase in the reflected power at $2 \mathrm{~mW}$ which indicates that the Brillouin threshold is reached. For comparison, we calculate the SBS threshold power using analytical equation in (7). The calculated analytical threshold power is $2.1 \mathrm{~mW}$ and this value is comparable with that obtained using the numerical method.

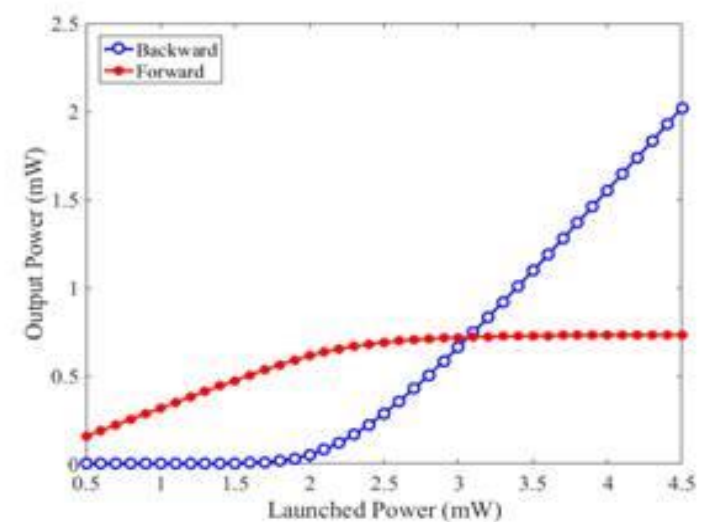

Fig. 2: Forward (transmitted) and backward (reflected) powers as a function of the input power launched into a 25-km single mode fiber (SMF)

Fig. 3 shows how the pump and Stokes wave varies along a $25 \mathrm{~km}$ long SMF when the pump is incident at $z=0 \mathrm{~km}$, while the input signal is launched at $z=25 \mathrm{~km}$. The evolution of the pump and Stokes wave are solved numerically using equations (1) and (2) with the $3 \mathrm{~mW}$ input pump power. Results show that the pump power degrades to $1 \mathrm{~mW}$ at $z=25 \mathrm{~km}$ which represents a $2 \mathrm{~mW}$ loss. On the other hand, the Stokes power rises to $0.7 \mathrm{~mW}$ at $z=0 \mathrm{~km}$, meaning only $35 \%$ of the pump power is transferred to the Stokes wave. It is worthwhile to note also that the power transfer occurs within the first $2.5 \mathrm{~km}$ of the $25 \mathrm{~km}$ SMF. This shows that the power transfer happens at the beginning of the SMF. 


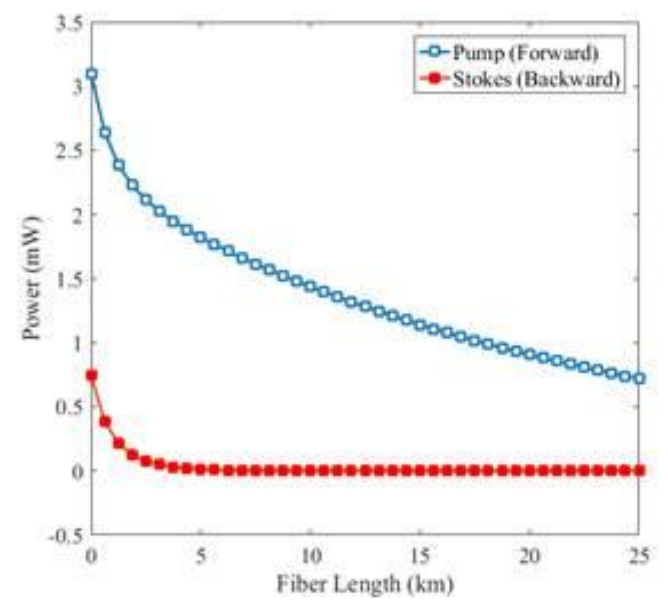

Fig. 3: Evolution of pump and Stokes powers along a 25km long SMF for pump power of $3 \mathrm{~mW}$

\section{Conclusion}

This paper has numerically investigated the behaviour and phenomenon of SBS effect in the optical fiber. Although SBS limits the channel power, it can be manipulated as a multi-wavelength laser source in optical communication systems. The results show that the Stokes wave experience rapid growth due to the transfer of power from the pump through SBS process. For further development, the polarization effect on the SBS threshold can be studied more thoroughly in order to enhance the efficiency of SBS-related applications such as fiber lasers and optical signal processing.

\section{Acknowledgements}

This work was supported in part by Research Acculturation Collaborative Effort (RACE) Vot 1509 and Fundamental Research Grant Scheme (FRGS) Vot 1622. Both grants are granted by the Ministry of Higher Education (MOHE) Malaysia.

\section{References}

[1] G. P. Agrawal, "Nonlinear Fiber Optics," in NewYork, NY, USA: Academic Press, vol. 4th ed, 2007.

[2] N. A. Cholan, M. H. Al-Mansoori, A. S. M. Noor, A. Ismail and M. A. Mahdi, "Switchable single- and dualwavelength erbium-doped fiber laser assisted by four-wave mixing with wide and continuous tunability," Appl. Phys. B, vol. 115, no. 2, pp. 251-256, 2014.

[3] K. Ogusu, "Analysis of steady state cascaded stimulated Brillouin scattering in a fiber Fabry Perot resonator," IEEE Photon. Technol. Lett., vol. 14, no. 7 pp. 947-949 July 2002.

[4] M. Ajiya, J. A. Oladapo, and N. A. M. A. Hambali, "Lasing threshold characteristics of multi-wavelength Brillouin-erbium laser in the L-band region assisted by delay interferometer," J. Nonlinear Opt. Phys. Mater., vol. 25, no. 2, p. 1650024, 2016.

[5] J. Tang, J. Sun, L. Zhao, T. Chen, T. Huang, and Y. Zhou, "Tunable multiwavelength generation based on Brillouin-erbium comb fiber laser assisted by multiple four-wave mixing processes," Opt. Express, vol. 19, no. 15 pp. 14682-14689, July 2011.

[6] N. A. Cholan, M.H.Al-Mansoori, A.S.M.Noor, A.Ismail and M.A.Mahdi, "Formation, properties and role of residual waves as seeds in multiwavelength Brillouin-erbium fiber laser,” Opt. Commun., vol. 329, pp. 163-167, 2014.

[7] R. Sanchez-Lara, J. A. Alvarez-Chavez, F. Mendez-Martinez, L. de la Cruz-May, and G. G. Perez-Sanchez, "Threshold and maximum power evolution of stimulated Brillouin scattering and Rayleigh backscattering in a single mode fiber segment," Laser Phys., vol. 25, no. 3, p. 35103, 2015.

[8] A. Kobyakov, M. Sauer, and D. Chowdhury, "Stimulated Brillouin scattering in optical fibers," Adv. Opt. Photon., vol. 2, no. 1, pp. 1-59, 2010. 